Article

\title{
User Monitoring in Autonomous Driving System Using Gami- fied Task: A Case For VR/AR In-Car Gaming
}

\author{
Joseph K. Muguro ${ }^{1,2, *}$, Pringgo Widyo Laksono ${ }^{1,3}$, Yuta Sasatake ${ }^{1}$, Kojiro Matsushita ${ }^{1}$ and Minoru Sasaki ${ }^{1, *}$ \\ 1 Department of Mechanical Engineering, Gifu University, 1-1 Yanagido, Gifu 501-1193, Japan \\ 2 School of Engineering, Dedan Kimanthi University of Technology, 657-10100, Nyeri, Kenya \\ 2 Industrial Engineering, Universitas Sebelas Maret, Surakarta, Indonesia; pringgo@ft.uns.ac.id (P.W.L) \\ * Correspondence: sasaski@gifu-u.ac.jp (M.S); Tel.: +81-58-293-2541; joseph.muguro@dkut.ac.ke (J.K.M.)
}

\begin{abstract}
Background: As Automated Driving Systems (ADS) technology gets assimilated into the market, the driver's obligation will be changed to a supervisory role. A key point to consider is the driver's engagement in the secondary task to maintain the driver/user in the control loop. The paper's objective is to monitor driver engagement with a game and identify any impacts the task has on hazard recognition. Methods: We designed a driving simulation using Unity3D and incorporated three tasks: No-task, AR-Video, and AR-Game tasks. The driver engaged in an AR object interception game while monitoring the road for threatening road scenarios. Results: There were no significant difference of means in the groups as per one-way ANOVA $(F(2,33)=4.34, p=.0213)$ suggesting little to no impacts on the task involvement. Game scoring followed three profiles/phases: learning, saturation, and decline profile. From the profiles, it is possible to quantify/infer drivers' engagement with the game task. Conclusion: The paper proposes alternative monitoring that has utility, i.e., entertaining the user. Further experiments AR-Game focusing on real-world car environment will be performed to confirm the performance following the recommendations derived from the current test.
\end{abstract}

Keywords: Autonomous Driving System; In-Car Gaming; Driver Behavior; Driving Related Tasks; $3 \mathrm{D}-\mathrm{VR} / \mathrm{AR}$

\section{Introduction}

Automation in vehicles has brought about a paradigm shift in the industry with much anticipated Autonomous Driving Systems (ADS) technology in the turn of the $21^{\text {st }}$ century. To date, driver assistance has attracted the interest of researchers and automobile manufacturers, as noted in the developed systems. Assistive technologies like emergency braking, lane-keeping, cruise control, and others have been deployed successfully [1].In the next decade, assistive technologies will give way to conditioned autonomous driving, a situation whereby the autonomous system takes over longitudinal (acceleration and deceleration) and lateral (steering wheel and turns) of the vehicle [2].

Automation levels are standardized by the society of automotive engineers (SAE) standardization ranging from SAE level 0 for no automation to SAE level 5 for full autonomy [3]. Interest to this paper is SAE level 3, 4, and 5, where the driver's obligation is primarily a supervisory one. According to the standard, as from level 3 , the driver is not mandated with constant monitoring of the driving environment but will need to resume control in case of unforeseen encounters. The system issues a take-over request when it encounters uncertainties (e.g., missing road markings, foggy weather). To this end, for automation levels, the driver/user of ADS would be free to engage with secondary tasks (Non-driving related tasks) during transit. 


\subsection{Non-driving related tasks (NDRT) and driving-related tasks (DRT)}

In a conventional vehicle system, NDRT encompasses all tasks (secondary tasks) engaged by the driver [4]. NDRT includes operating handheld devices, operating in-car systems, communicating with passengers or on calls, among others. On the other hand, driving-related tasks (DRT) encompass tasks performed by the driver to aid in the entire driving process [5]. DRT may include tasks like checking the speedometer, monitoring the side-view mirror, amongst others.

Up to date, research and policies have been focused on dissuading drivers from engagement in a secondary distractive task(s) owing to the threat these activities pose both to the driver and other motorists [6]. Research has been conducted to understand driver behavior in an NDRT environment for ADS. A paper by [4] investigated the effects of NDRT on the quality of take-over in varying traffic situations. The authors employed two tasks: visual surrogate reference task as a representative of eyes-off-road and n-back test as a mind-off-road engagement. There was no significant reported difference between the two types of distraction. A paper [7] evaluated the influence of drivers in news and email reading, watching a video clip, and engaging with a tablet. Another paper [8] used video and a tablet gaming NDRT to evaluate driving behavior in a critical conditional take-over. The authors concluded that there was no influence of NDRT on reaction time. Authors [9] found that engaging in distractions can reduce up to $27 \%$ drowsy tendencies in the automated drive. From the standpoint of embracing the potential usage of distractions to enhance safety, system designers' focus is to develop a system that maximizes transitioning from distraction to resuming control.

With the current technological advancement, the driver can be engaged in a myriad of activities, each soliciting the driver to different states. To reduce the chances of failure in a take-over, authors [9] argue that ADS will necessarily be tasked with monitoring the driver to assess the readiness to take over control. One way of achieving that is monitoring the task the driver is undertaking. Authors [10] argue that engagement with gamification in driving can reduce the risks associated with boredom and reduced vigilance. With this in mind, we have conceptualized a driver engagement model based on the content source and management routines, as shown in Table 1.

Table 1. Driver engagement model.

\begin{tabular}{|c|c|c|}
\hline State & Engagement status & Description \\
\hline & & No distractions \\
\hline 0 & Monitoring state & $\begin{array}{l}\text { The challenge with this level is that it is hard to maintain a } \\
\text { no-distracted status for an extended period due to monot- } \\
\text { ony in ADS }\end{array}$ \\
\hline 1 & ADS Managed Tasks & $\begin{array}{l}\text { The driver engages with tasks like watching movies, games, } \\
\text { etc., that are managed by the ADS system. This gives the } \\
\text { advantage of the ease of passing relevant drive information } \\
\text { and an indirect driver monitoring system. }\end{array}$ \\
\hline 2 & External Devices Tasks & $\begin{array}{l}\text { The driver engages with tasks with connected devices } \\
\text { (smartphones, tablets, etc.) }\end{array}$ \\
\hline & & This allows for active sharing of relevant information \\
\hline 3 & Passive State & $\begin{array}{c}\text { The driver engages with tasks unrecognized to the system. } \\
\text { This covers all tasks, including unconnected devices and } \\
\text { naps }\end{array}$ \\
\hline
\end{tabular}

The desirable state is for the driver to be non-distracted and actively monitoring the road (monitoring state). Since this state is hard to maintain, we conceptualize three other states. In the primary level, the NDRT content is managed by the vehicles (ADS), i.e., start, stop, pauses interrupt, and other probes to focus the driver's attention. The foreseeable advantage of this is that information delivery can be optimized to integrate with the current road conditions. On the secondary level, external personal devices are linked to the system such that interrupts can rely on pertinent information instead of the driver having 
to build his/her situation awareness. Several authors [7,11,12] have investigated NDRT at this level. The third state is the passive one, with unconnected devices or tasks blind to the system like a deliberate nap. A passive level will be the ultimate experience of an ADS in level 4 and above.

Since ADS will eliminate the need for active driving inputs and constant monitoring of the road, activities performed by the driver will not be categorized as a distraction [9]. This is the paradigm shift modulated by automation, where distraction is desirable in a car environment, i.e., the DRT concept. As noted by the report [5], DRT can be a potential source of hazard in conventional driving. However, as ADS takes full shape, driving will be the distraction as roles get reversed. DRT in ADS is redefined to migrate from the conventional potentially hazardous task to a positive engagement that enhances the driving experience. In this case, DRTs seek to aid/promote overall improvement in the driving experience. To this end, activities that promote proper sitting posture, adherence to the proper hands-on-steering wheel, road monitoring, and leg-pedal positioning would be considered DRT. Intuitively, tasks that promote road monitoring and the hands-on steering wheel would improve the quality of taking over.

Fatigue is expected to set in quickly in autonomous cars than in conventional driving owing to reduced engagement $[13,14]$. Research by [11] reported that drivers in simulated automated cars showed physical signs of fatigue after 15-30 minutes of driving compared to manual drivers with longer endurance. The motivation for studies directed towards gaming stems from the fact that, in long-distance journeys, drivers and passengers alike are confined with few interactivity options. During transit, blank stares into the window, restlessness, impatience, and other experiences have happened to many travelers [15]. A remedy to this has been the use of recreational activities allowable by the mode of traveling [16]. With the introduction of automation, gaming can be explored in a car environment without compromising the safety of stakeholders.

\subsection{Related works and current state of automation}

This paper focuses on gaming as a lucrative engagement that will be entertaining and an indirect environment contextualization scheme. We propose to monitor the driver's engagement as described in the literature with video and gaming tasks. This is an intuitive distraction that can keep the driver engaged. We found few works geared towards this line of inquiry. Similar research is reported in [17] focusing on a cross-car multiplayer game for the driver in level 3 and higher of an ADS. The authors designed a fully immersive multiplayer game for the driver, assuming full heads-up display and ad-hoc vehiclevehicle communication for co-located vehicles. A Cooperative in-car game is proposed in [18], targeting cooperation between parents and passenger kids using handheld devices. Another paper [19] employed VR to an actual moving car with rendered underwater scenarios meant to offer a restful/mindful driving experience. While [20] used a similar moving vehicle with a VR system to render a flying (helicopter) environment with shootable game objects. One foreseeable challenge that is bound to affect the driver, who becomes the new passenger in ADS, is motion sickness [21,22]. An in-car game can remedy this by optimally synchronizing the experiences in the moving car and experienced content. This is the strategy proposed by holoride $\AA$, VR ride, and others $[23,24]$. Other researches have focused on using handheld gadgets, tying game experience to geo-locations, passengers, and others [18,25-27].

In the recent past, cars are being manufactured with inbuilt games, a very new concept, albeit it being played on a parked car. To this end, manufacturers like Tesla®, Mercedes-Benz ${ }^{\circledR}$, and others are paving the way for the future of gaming in vehicles. Tesla introduced the first game in cars (Atari games) playable on the screen [28]. In early 2019, Mercedes-Benz ${ }^{\circledR}$ introduced a video game (Mario Kart) on the center screen $[29,30]$. The deployed games are principally targeting a parked vehicle, thus enabling the car steering wheel and pedals. As a development to the parked-car games, Audi ${ }^{\circ}$, holoride $₫$ and Disney team released an in-car VR experience focusing on passengers in transit [23]. The 
game integrates (meshes) with car motion to deliver synchronized content in the VR world which is argued to relieve carsickness. This and other developments will further solicit games in cars targeting different in-car users.

Automakers have tested several concept cars with different features. Two of such concepts of particular interest to this paper are Zoox®, an Amazon-owned robot-taxi, and Chevrolet Env 2.0® [31,32]. The concept cars featured an overhaul to the conventional interior design with a notable elimination of the current infotainment system. This implies that an alternative information/entertainment system is needed that best suits ad and supports the new driving experience.

The current paper and the previous research focusing on gaming in a car have been predominantly prototypes and proof of concepts. The general objective has been timefiller, dislocation of cyber-physical space, immersive gameplay, and exploration of new entertainment gaming concepts. Time-filler emerges from the monotony of driving with limited engagement activities. Cyber-physical dislocation applies to individuals who prefer to be in a different (virtual) environment instead of a congested public transit system, as discussed by [33]. The proposed gaming approach, a form of human-machine interface, can sustain vigilance in ADS and increase information flow by aiding visual attention using gamified tasks.

\subsection{Present study}

The overall objective of the paper is to study driver behavior in an ADS with gamified tasks in a 3D VR simulated driving environment. As previous studies have shown, boredom in an ADS, particularly in a monotonous route, would be far worse than a conventional driving experience [10]. With the introduction of an AR, users would be presented with new content overlaid in any route, making the journey enjoyable and less tedious. We propose a game designed to be played by the driver of an ADS during autonomous mode. The game interactions are simple to avoid over-immersion at the expense of environmental awareness, as recommended by [18]. Additionally, gaming elements appear within the driving region of interest. This will assist indirectly in road monitoring and increased Situational awareness.

Several researchers identified awareness in driving as key to safe driving [34-36]. At the onset of level 3 automation in ADS, concerted efforts will be needed to maintain the driver's visual search path to the driving environment. The reported cases of traffic incidences involving self-driving cars have partly been due to the failure of the safety driver being disconnected from the environment $[37,38]$. As ADS develops, there will be perfect system performance, but passengers and drivers alike will need to be aware of the developing situation on the road until then. One such way is the use of un-obstructing AR games to guide vision to emerging/developing situations.

From [3], a fallback-ready user should be receptive to requests or eminent vehicle system failure whether a take-over request is issued or not. This calls for sustained vigilance on the driver's side, which is the focus of this paper. As such, the current research is concerned with the following:

1. Investigate how well the driver can recognize threatening driving scenarios while engaging in a game. This will be indicated by the time taken to press the designated button (recognition time) accurately.

2. Evaluate drivers' engagement with the task using score profiles to make inferences on the drivers' state. Game scores will be used as an indirect measure to infer engagement in the task and, by association, vigilance.

3. Investigate driver's interaction with the virtual environment. Based on the interaction model, the driver may be overly engrossed or disinterested in the task. Eye data will be utilized to identify trends in gaze behavior to confirm road monitoring. The gaze trends will be used as design recommendations for indirect monitoring systems. The contribution of the present study is an investigation on the use of VR and headmounted display as an alternative in-car infotainment system and characterization of 
driver state using gaming modalities. A business case for level 4 and above is well captured by [39], where engagement state and visual attention metadata captured is applied in tourism. As a pilot study, the current paper seeks to shed light on design considerations for infotainment systems. The findings will be applied in a real-world environment with a moving car to study various aspects of entertainment in an automated vehicle.

\section{Materials and Methods}

\subsection{Driving simulator}

We designed a custom car simulator scene with a Unity3D game engine for driver analysis. A virtual car is configured on a rule-based autopilot (use of waypoints) to mimic autonomous drive in the configuration. Thus, no control inputs were required from the user. The vehicle was designed to move in a straight path (along the z-axis) with minimal speed variation and turns. A rural terrain was adopted with a two-lane asphalt road and minimal terrain details. The scene was designed to be monotonous with only road markings, greenery (grass) terrain all through on a clear blue sky. This was done to dissuade the user's gaze from wandering to terrain details but rather focus on the gaming relevant objects (popup traffic and game elements). Fove ${ }^{\circledR}$ 3D Head Mounted Display (HMD) was used for VR content rendering in the prototype game. The simulation was run on a Windows 10 PC with an Intel® Core i7 processor and GeForce GTX 1070 graphics card.

In the design, we included staged popup traffic events to assess the driver's threat recognition time. Popup events appeared in random order at a predetermined location (location A, B, and C) shown in Figure 1. Figure 2 shows sample scenes with popup traffic and game elements. Popup traffic appeared after every $2 \mathrm{Km}$ in a total of $25 \mathrm{Km}$ distance covered cruising at a constant speed of $30 \mathrm{~m} / \mathrm{s}$. Twelve (12) popup instances were displayed for each experiment. The entire drive-path region of interest covers the pavements and the two lanes, as shown in Figure 1.

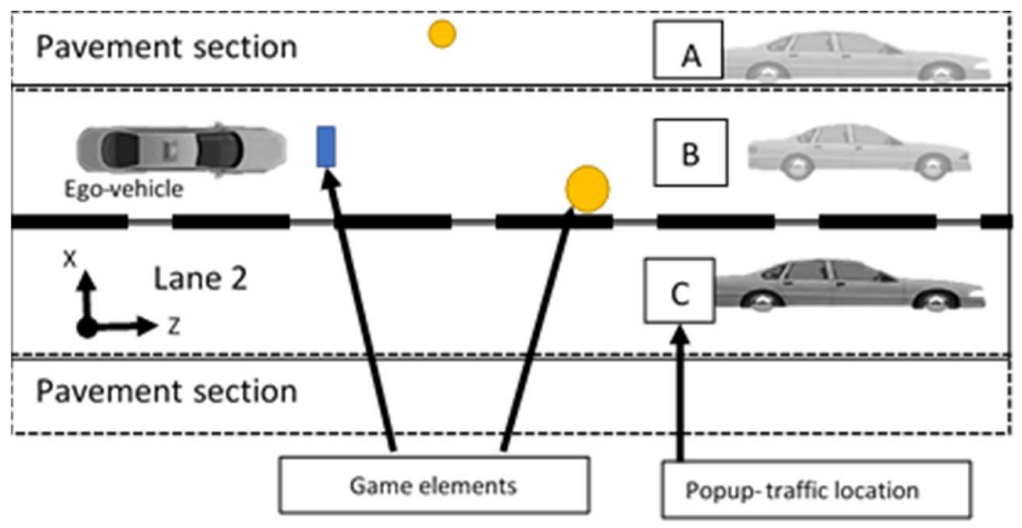

Figure 1. Simulation scene setup showing popup traffic location and conceptual game elements. The experiment is conducted in a two-lane road section with pavement on both sides as indicated. 


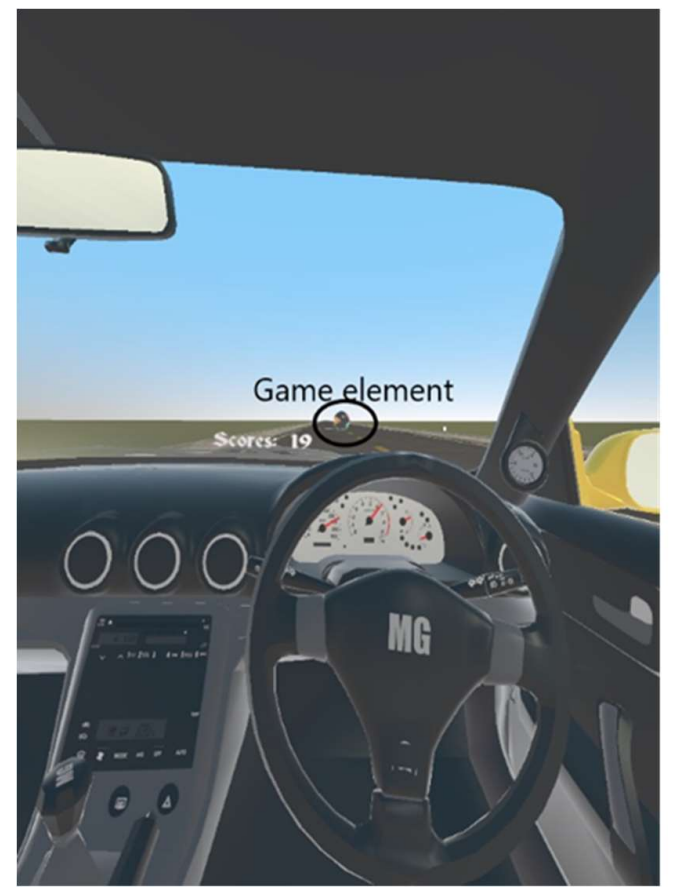

(a) AR-Game elements view

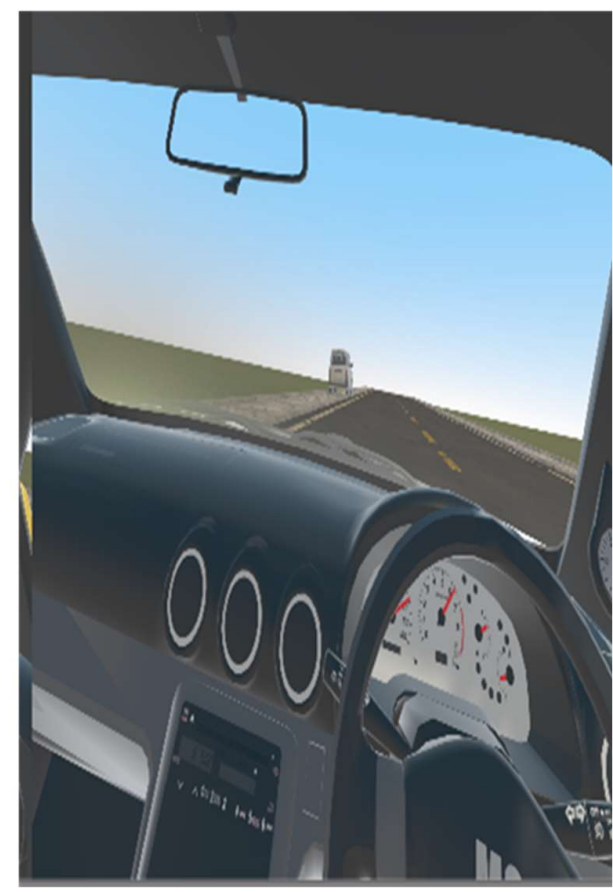

(b) Popup traffic vehicle

Figure 2. Sample VR scene in the driving simulator.

\subsection{Game mechanism design}

The paper proposes introducing gaming in a car as a pass-time activity that will improve engagement and offer entertainment. The driver is actively engaging with elements on the road while monitoring traffic on the game setup. A controllable paddle object (player) is located a few meters from the cars' position, which is visible by the driver. The paddle slides along the $\mathrm{x}$-axis and is translated by the position of the car (z-axis). As the car moves autonomously, collectible objects are spawned ahead on the drive path by the gaming engine, as shown in Figure 2(a). The spawned objects appeared at an interval of 2 seconds.

When the controller paddle (Unity 3D game object) collides with the mesh of the spawned object, a score is registered (intercepted) and the contrary for a missed object. This is made possible by Unity-3D's physics system that checks for the interaction of game objects. Missed objects will be recorded alongside the spawn point to analyze the scoring profile of the position.

The driver moves the paddle position to intercept AR-Game spawned objects (elements) using a physical paddle controller shown in Fig 3. The player increases points upon a successfully intercepted object. In the case of a missed object, a penalty is executed (decrement in displayed score), and data is recorded for further analysis of the missed object profile. Score progress is logged and displayed in the dashboard of the car. Gaze information was recorded using the inbuilt eye-tracking system of FOVE® HMD. The data is logged together with car position and game score progression for every frame at a sampling rate of 65-75 frames per second.

We propose to set up Video on HUD so that the driver can transition from video to road monitoring effortlessly. This is achieved by projecting the contents in a HUD type of screen instead of having the video player located below the driver's view in a cockpit, as is the case in current car design. With this setup, the video is rendered in the upper part of the windscreen, leaving enough window for road monitoring. The setup is meant to reduce the time taken to have eyes on the road.

\subsection{Experiment setup}


The subjects sat comfortably in an office chair with the camera view inside the car positioned in a typical driver seat in a 3D-VR environment. Figure 3 shows a test subject using 3D VR and steering wheel input controls for the game. We used the Thrustmaster ${ }^{\circledR}$ steering wheel attached to the PC running the simulator for popup object buttons and game controller. The subjects were given a test scene of about 5 minutes to familiarize themselves with the control protocols. When fully mastered, a logged experiment was conducted ranging between 20-25 minutes.
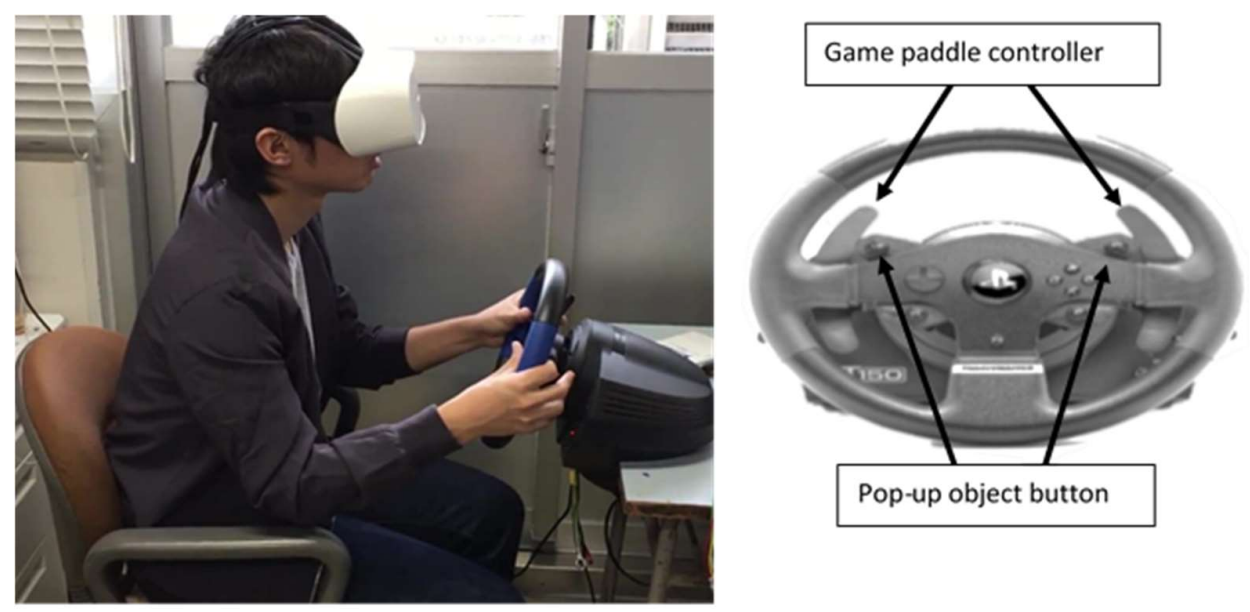

Figure 3. Test subject with 3D VR Head mount display and driving steering wheel setup.

The user was expected to recognize the threat and push a corresponding button on the steering wheel shown in Figure 3 as a popup object button. No auditory warnings were issued when the traffic appeared. Therefore, the driver relied on a visual search to identify relevant information from the drive path and react accordingly. We experimented with the user engaged in a game and compared the threat scenario recognition time to a case where the user has no or mild physical engagement (i.e., watching a video clip or notask). The time taken (recognition time) to detect threats on the road is expected to be impacted by the task or the driver's loss of attention. Interaction with the AR elements is evaluated in the form of game scores and gaze information.

\subsection{Participants}

Students comprised the participants in the study and were recruited following approval from Gifu University ethics committee. Thirteen subjects took part in the experiment with an average of 26.6 years (std 6.2) from different nationalities. Real-life driving or gaming experience was not considered in the current study. A preparatory drive scene was presented before the recording of data. In this test, the subjects were introduced to the controls and buttons and the general objective of the experiment.

The participants were divided into two groups, the control group ( 3 subjects) and the test groups (10 subjects). The control group was presented with the tasks in random order, while the other group was presented with gaming tasks to avoid pre-exposure. Each subject's gaze information, button presses, scores, and interaction with game elements are logged in an excel file for further processing. The experiment lasted between 20-30 minutes per subject. No incentives were offered to the subjects. Data analysis was performed using Matlab® software. 


\section{Results}

\subsection{Recognition (reaction) time}

\subsubsection{Comparison of tasks}

To compare the performance of recognition time with different tasks, the control group tried the three tasks in random order. The performance is evaluated as shown below. Figure 4 shows a boxplot of the recognition time recorded in all the popup cases with or without a DRT. The evaluated tasks: No-Task, Game (AR-Game), and Video, had a recognition time difference of less than $1 \mathrm{~s}$. for the subjects. Outliers in a No-Task scene represent instances where the driver was not paying attention to road events. The figure shows a case where driving with AR-Game would have slightly slow recognition time with an advantage of consistency (a compact interquartile range).

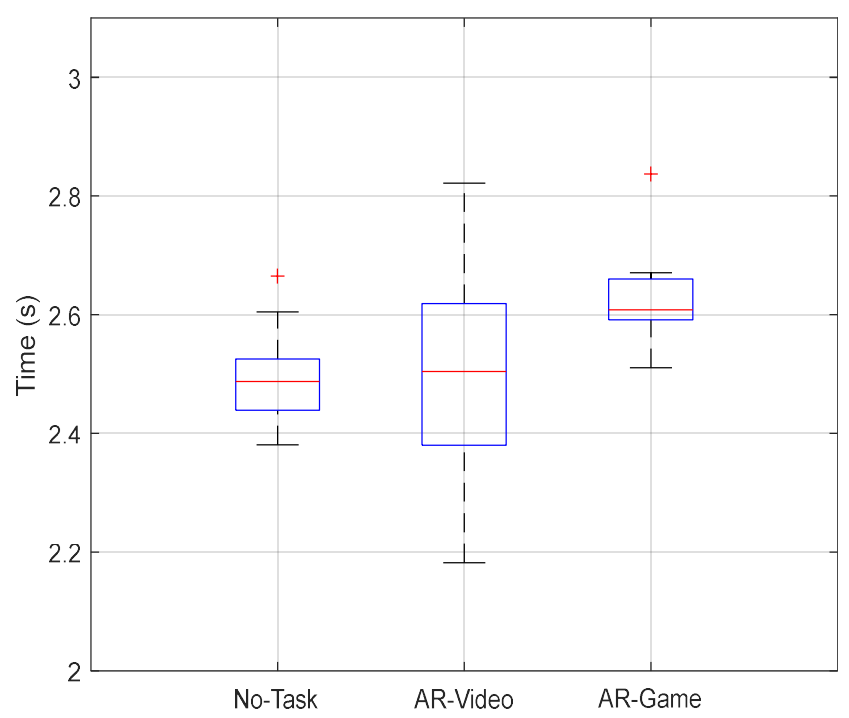

Figure 4. Recognition time of driver with different engagement.

There were no statistically significant differences between the means of the groups as reported by one-way ANOVA $(\mathrm{F}(2,33)=4.34, \mathrm{p}=.0213)$. From the figure, driver in NoTask had the best recognition time $($ mean $=2.492$, std $=0.083)$ and AR-Video (mean $=2.506$, std $=0.179)$. AR-Game, (mean $=2.627$, std $=0.081)$ was the slowest as expected. In No-Task and Video tasks, the user's hands are not occupied with any activity and as such, pressing of buttons is instantaneous immediately traffic pops up. In the case of an AR-Game, the user is actively controlling a gamepad and takes more time transitioning to button press from active game control. A noticeable difference is an interquartile range (IQR), which was found to be 0.09 , 0.2, and 0.07 s. for No-Task, AR-Video, and AR-Game tasks, respectively.

\subsubsection{Gaming recognition time}

The focus of the study was to investigate the impacts gaming would have on the reaction time. Figure 5 shows the boxplot of the recognition time for 10 of the users evaluated in the experiment. In all the cases, the drivers reacted within 2 seconds after popup traffic. The data captures a case where the driver did not press any button within the time. This is shown as an outlier in driver no. 7. In the plot, the line represents the median, and the boxes are the interquartile ranges (IQR) of the 12 popup instances. 


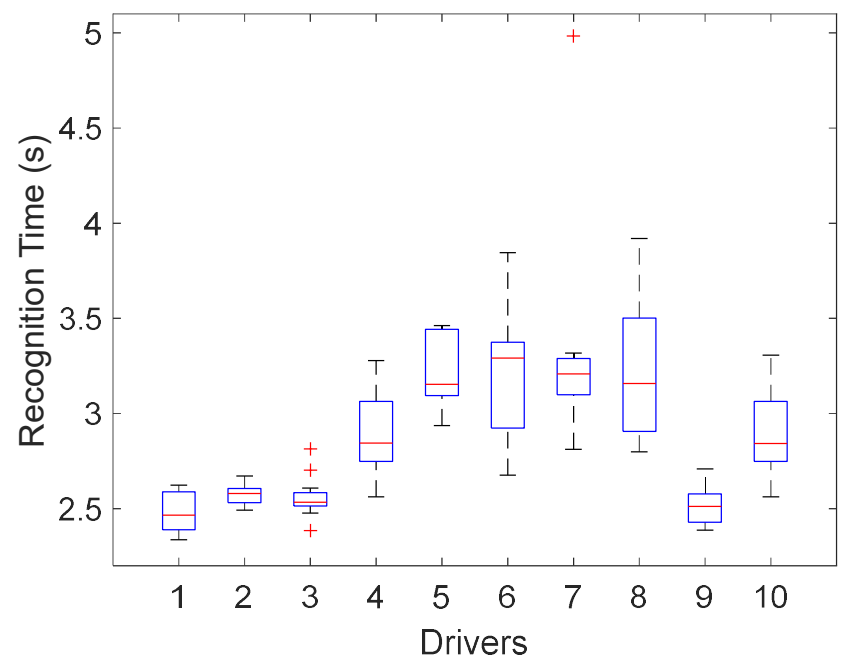

Figure 5. Overall AR-Game reaction time for different users.

\subsection{User gaze tracking}

As mentioned, the experiment was inwardly recording eye gaze as the user interacts with both popup and game elements. The results below show gaze direction progression overlaid onto the conceptual scene setup shown in Figure 1. This is done to reveal tendencies of fixation or scanning the environment around the objects of interest. Gaze direction results are as shown in Figure 6. The figure shows scatter plots of gaze direction (x-axis) of the driver, 5-7 seconds before a button-press. The $x$-axis of the drive path (region of interest) ranged between -5 and 5 from the center view in the figure. Center view (zero points) represents the position the gaze would make in a VR environment if the driver looked straight into the environment. From the result, the user's gaze is actively engaged in the road environment both on the left and right side of the travel lane. The strength of the scatter plots (concentration points) reveals gaze fixation points, instances when there is an object of interest in the scene, while weak scatters are during gaze movement (scanning of environment or transitioning to the next object). Two distinct patterns in the gaze information are identified as localized search and scanning patterns.

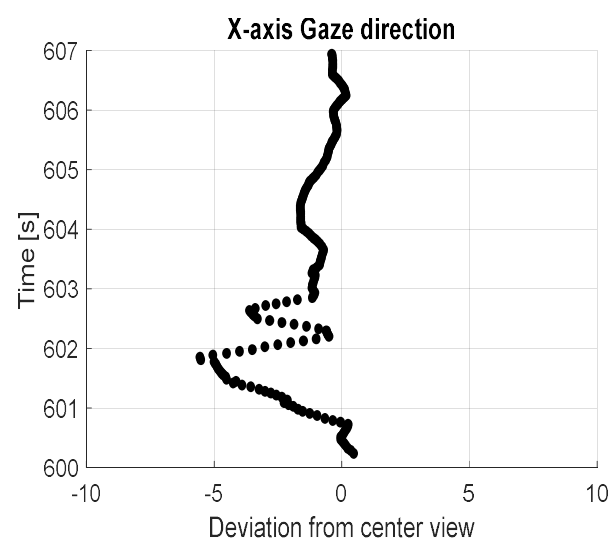

(a) Localized gaze fixation (focused user's visual attention)

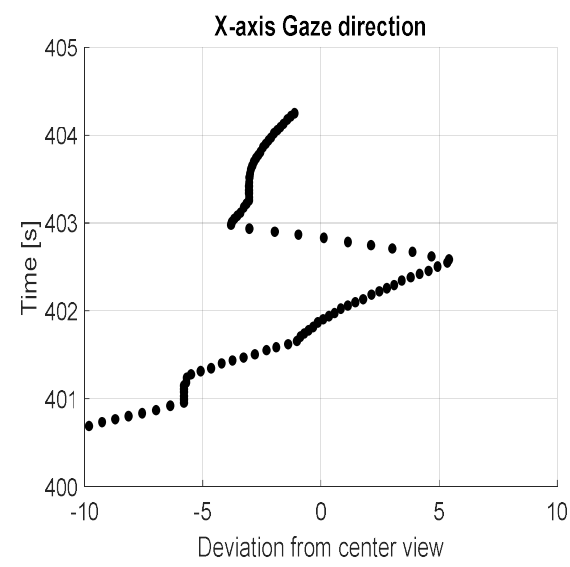

(b) Gaze scanning of the drive path

Figure 6. Gaze information in different visual search scenarios.

\subsection{Score profiles}




\subsubsection{Intercepted objects profile}

Figure 7 shows the intercepted AR-Game elements (score) distributed in a 100-second interval (segment) from the start to the end of the simulation. Each duration represents a period in which 50 elements were spawned in the driving scene, and the score is the cumulatively spawned objects. The accumulated scores have a direct relation with user game interactions. After initial learning of the basics, the scores are expected to rise to a level allowable based on the user's hand coordination skills. The results show a general profile of scoring progression, what we are referring to as intercepted objects profile. Three distinct profiles/patterns were observed: a learning phase (positive gradient), saturation (constant gradient), and a decline (negative gradient). This is highlighted in Figure 7(a)-(c) using trend lines. An intermediary stage of saturation was also noted but is subsumed in Figure 7(b) during the transition.

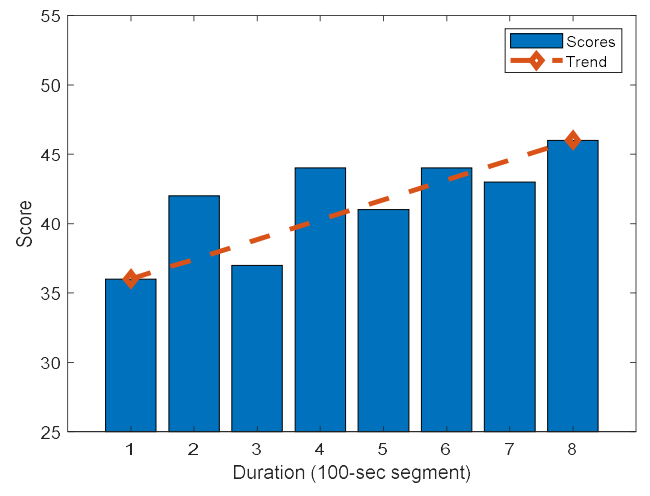

(a) Positive gradient

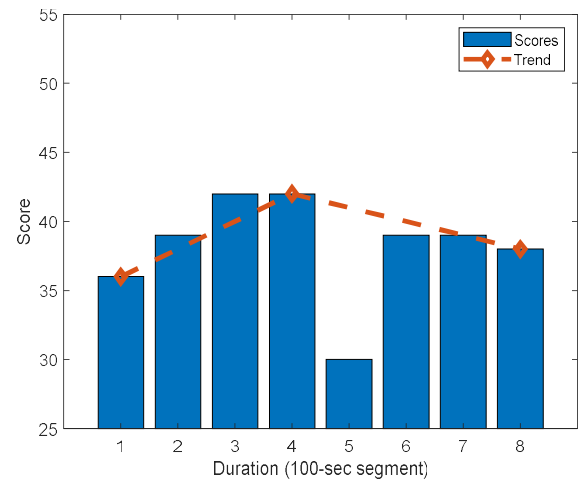

(b) Positive/Negative (decline) gradient

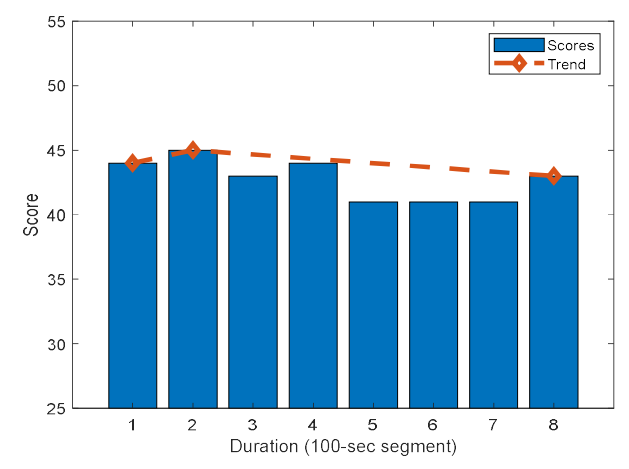

(c). Saturation trend

Figure 7. Score progression and trends for different intercepted objects.

The phases are generated after observing the data from all users. Extra testing of subjects conformed to the three phases and did not provide new information. The significance of each of the profiles is discussed in a later section. Figure 8 shows the percentage score of the drivers for the entire course. The figure shows an average scoring of $83.4 \%$, verifying the game's playability to a satisfactory level. 


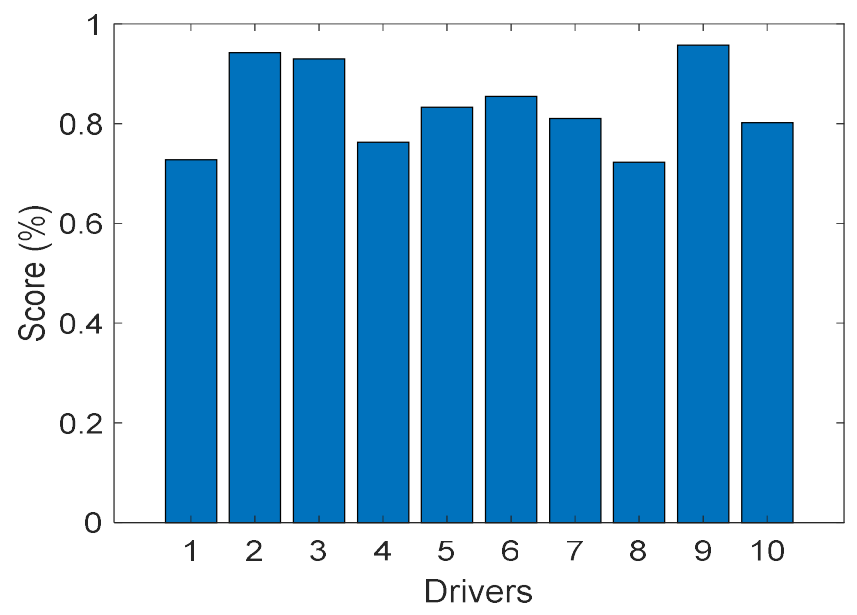

Figure 8. Overall intercepted objects (Scores).

\subsubsection{Missed objects profile}

Like the intercepted objects, missed objects are equally significant as a pointer of the reasons for a missed object/error. As with any gaming engagement, the rules for losing are equally essential and telling. In the game, a missed object is reported when the paddle did not intercept the game element. Missed objects profile is formulated by plotting deviation index (distance from paddle to the target game element). This information is also tied to the respective spawn point to formulate a profile. The deviation index is calculated as shown in Eq. (1) below.

$$
I_{\text {dev }}=\mid \text { missed_obj } j_{i}-\text { paddle_pos } \mid
$$

where $i$ represent the spawn position 1:5, Paddle_pos, in this case, represents the current position of the paddle, and missed_obj represents the position of the currently missed object.

Deviation index, Idev is given as the absolute separation distance between the paddle and the spawned AR-Game element. For each of the spawn points 1-5, the corresponding average deviation index is logged and is shown in Figure 9. The deviation increases as the object move from the middle "U-shape" around spawn point 3 (drivers center view). The deviation index standard deviation error also increases from the center spawn point.

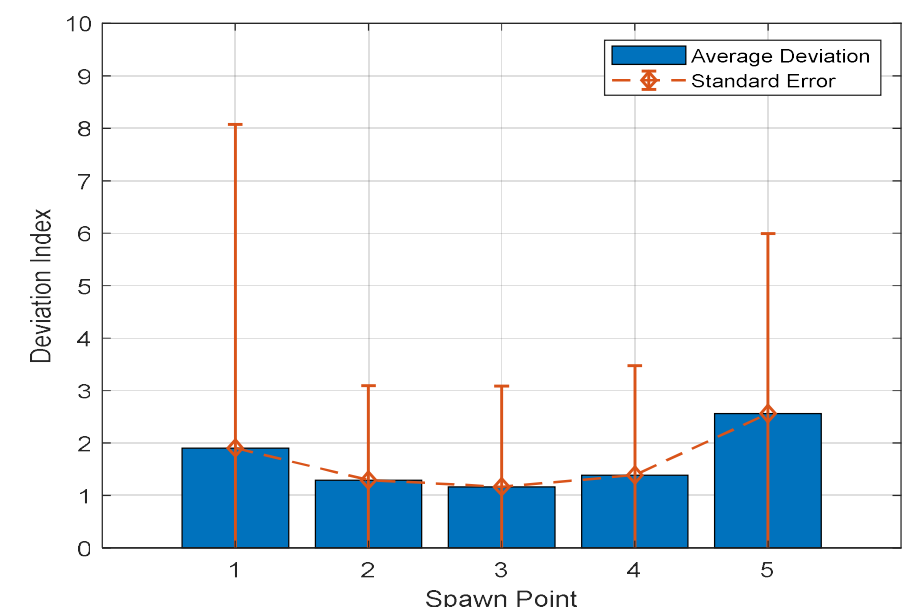

Figure 9. Missed objects deviation index.

\section{Discussion}


This paper aimed to investigate the use of games in an autonomous car environment. To this end, we sought to gather driver behavior and tendencies to infer the engagement and driver state level. The investigation sought to answer the question of what the effects will be of engaging in a simple controller game in an ADS environment. We designed an AR game inside a driving simulator to analyze driver's engagement with game elements and staged popup traffic. Similar research had no agreed-upon standard of reference or evaluation scheme with more emphasis on the design of approach [10,17,26,27].

\subsection{Recognition time and visual search}

In the experiment, recognition of popup objects was compared between three engagement levels: no task, watching a video clip, and gaming tasks. The results are shown in Figure 4. From the ANOVA tests, the means for all the tasks were not statistically different $(p=.0213)$. An important observation is the interquartile ranges of the tasks evaluated. The tasks had an IQR of 0.09, 0.2, and 0.07 s for No-Task, AR-Video, and AR-Game, respectively. This suggests that the AR-Game driver has a consistent RT compared to other tasks that fluctuate with attention shifts.

The overall recognition time for all subjects yielded a reaction time of $2.9 \mathrm{~s}$, which agrees with the findings of other researchers $[8,40]$. Recognition time is a general interest for automobile system designers and researchers alike in intention studies. Wenxiang et al. described the process and mechanism in intention recognition as having three parts: successful detection, correct recognition, and timely response [41]. The findings suggest that a driver engaging in an AR-Game would not be impaired by the gaming elements in recognizing threatening scenarios.

Here, we explored one of the advantages of AR-Game, focusing on the visual-search and patterns of the driver to different sections of the road. From Figure 6, we identified two visual search patterns: scanning and localization of the visual search. As objects are populated in the drive path (popup traffic and game objects), users' gaze reacts to each item. If the objects appeared within the same region, there was gaze fixation compared to dispersed gazes while scanning. The two patterns identified above represent cases where the user shifts his/her focus to interact with objects. Authors $[42,43]$ reported that the driver's gaze is dispersed in the environment compared to manual driving for ADS. As opposed to a dispersed gaze, the results presented in the paper follow a systematic and focused transition from one object to another.

We confirmed positive tracking of AR elements that can be applied in refining the users' gaze to relevant information along the drive path. At the advent of self-driving cars, highlighting personalized content that the user is most intrigued by or relevant information outside of the car environment, such as traffic signs, would be an added advantage. Researchers have linked gaze wandering with boredom and lost interest [44]. In this paper, we have only tracked the progression of the gaze to ascertain the way users tracked objects. Further analysis would be needed to ascertain cases of lost interest in the gaming activity using gaze information.

\subsection{Score profile as an engagement model}

We managed to introduce and control gaming elements in the driving path to reduce the monotony in the driving scene. The overall score for the drivers is as shown in Figure 8. The overall percentage score represents individual control skill which reflects the ease of game control and interaction. All drivers had an average score of $83.4 \%$ and a minimum of $72.3 \%$. Game score progression was theorized to reflect the user's engagement which is related to driver state. Considering the monotonous scene employed in the setup, the driver is only engaged with game-control or road monitoring. From this, missed objects will arise from loss of focus or shortfall in hand coordination skills. Consequently, as the participant engages in-game control, the progression trend will highlight the participant's engagement level. The intercepted object trends in Figure 7 show how the user interacts 
with gaming elements and indicates engagement as either enthusiasm, saturation, or declining interest.

Considering this is a game the users have never encountered before, a learning phase is expected at the onset, followed by either a decline or sustained scoring depending on the user's impression of the game. The trend curves derived from the data from different users are interpreted as follows. A positive gradient trend is indicative of an aspect of learning as the game progresses. This was present in all the drivers new to the simulation. As they learned the control rules, a saturation pattern was formed. The saturation trend would indicate users who have learned the control scheme and actively perform at personal peak allowable within the constraints. This level was sustained in cases where the user enjoyed the game or gave way for a downtrend. A declining trend was reported in most final stages of the simulation. A transition from positive to negative gradient would be an ideal place to introduce levels and or other gaming elements to keep the user engaged. If fed to the system, the trends identified in the result would add to the pool of feedback information of the current state of the driver. This way, the system will have a form of contextualization of the state of the driver.

\subsection{Game design consideration}

As noted from Figure 9, AR-Game elements on the sideways were easily missed compared to center objects. This agrees with the results from different authors on the anisotropic perception of object size, distance, and positioning in a VR environment [17,45]. From these results, the deviation index increases as the position of the playable object depart from the center. The "U-shape" error pattern is a critical consideration in the enjoyment/challenge of AR elements. It points to the hurdle that must be considered in terms of $3 \mathrm{D}$ world reconstruction due to visual perception and biases in the human brain. Careful consideration of the playable/interact-able environment is recommended.

\subsection{Limitations}

The experiment employed a 3D-VR game prototype in place of an actual driving environment. A real driving scenario would be preferable, but the ADS technology is not fully matured; a substitute of VR has been utilized to give insights and design for future development. The limitation in this has to do with the lost sense of danger which might impact the generalization of the recognition time. However, intuitively, in a real threat scenario, threats would be processed with higher priority, not lesser. Motion sickness manifesting as mild eye fatigue was reported by one subject, but the greater majority did not have any physical discomfort. The test subjects comprised a relatively young population. In the case of older test subjects, the effects of VR usage might be more pronounced.

The design of the experiment is also not exhaustive; as a proof of concept, the study was conducted with limited test subjects, targeting university students in a controlled environment. Additionally, the setup considered a traffic scenario with no competing stimuli. Further investigations will be conducted incorporating diverse groups with a variety of stimuli in a real-world environment. Studying behavior in a real-world environment with near-natural stimulation of physical car movements, acceleration, braking, and other dynamics, will offer important information for the design of automated vehicles and infotainment systems. In the future, the 3D-VR/AR game will be tested in an actual moving car following the recommendations derived from this test.

\section{Conclusions}

In conclusion, this paper has explored what the driver will be engaged with for SAE Level 3 automation and above. The proposed scheme uses VR game tasks that add value to the automation system instead of just entertaining. In this paper, the driver engagement model identified as ADS managed content has been evaluated. The proposed scheme is the use of AR-Game that meshes with the driving scene. During the driving scene, the user was presented with popup traffic to evaluate recognition time. We found little to no 
effect on recognition time when drivers engaged in an AR game from the experiment. In the advent of ADS systems, secondary tasks will be needful to add value to the driving experience and maintain vigilance.

From the discussion, driver monitoring through score progression on the road environment by gaming modality has been achieved. Learning, saturation, and decline profile were identified as the prominent trends that would be useful in contextualizing the engagement model. When the ADS manages the content, it will be possible to inference the driver state with no adverse effects on recognizing threatening driving scenarios.

Gaze information results suggested that it is possible to focus drivers' visual attention and tie it to a relevant source of information using game elements. The results confirmed the anisotropy of objects in a 3D environment, as seen in the missed object profile. This is a design feature that should be considered when designing in-car games that have 3D interactivity. Further research should be conducted to understand driver's behavior in a multi-stimuli environment and various gaming options. The overall findings indicate that gaming-in-car would be advantageous with negligible impacts on the road monitoring performance where such is needed.

Author Contributions: Conceptualization, Joseph Muguro, Kojiro Matsushita and Minoru Sasaki; Data curation, Joseph Muguro; Formal analysis, Joseph Muguro, Kojiro Matsushita and Minoru Sasaki; Investigation, Joseph Muguro, Pringgo Laksono and Yuta Sasatake; Methodology, Pringgo Laksono and Yuta Sasatake; Software, Yuta Sasatake; Supervision, Kojiro Matsushita and Minoru Sasaki; Writing - original draft, Joseph Muguro; Writing - review \& editing, Pringgo Laksono, Kojiro Matsushita and Minoru Sasaki. All authors have read and agreed to the published version of the manuscript.

Funding: This research received no external funding

Institutional Review Board Statement: The study was conducted according to the guidelines of the Declaration of Helsinki, and approved by the Gifu University ethics (approval procedures number 27-226) issued by Gifu University ethics committee).

Informed Consent Statement: Informed consent was obtained from all subjects involved in the study.

Conflicts of Interest: The authors declare no conflict of interest.

\section{References}

1. J. M. Anderson, N. Kalra, K. D. Stanley, P. Sorensen, C. Samaras, and O. A. Oluwatola, "Brief History and Current State of Autonomous Vehicles," Auton. Veh. Technol. A Guid. Policymakers, pp. 55--74, 2014.

2. S. K. Jayaraman et al., "Pedestrian Trust in Automated Vehicles: Role of Traffic Signal and AV Driving Behavior," Front. Robot. AI, vol. 6, p. 117, Nov. 2019, doi: 10.3389/frobt.2019.00117.

3. S. Standard, "J3016B: Taxonomy and Definitions for Terms Related to Driving Automation Systems for On-Road Motor Vehicles - SAE International," 2018. [Online]. Available: https://www.sae.org/standards/content/j3016_201806/. [Accessed: 17-Dec-2020].

4. J. Radlmayr, C. Gold, L. Lorenz, M. Farid, and K. Bengler, "How Traffic Situations and Non-Driving Related Tasks Affect the Take-Over Quality in Highly Automated Driving," Proc. Hum. Factors Ergon. Soc. Annu. Meet., vol. 58, no. 1, pp. 2063-2067, Sep. 2014, doi: 10.1177/1541931214581434.

5. T. a. Dingus et al., "The 100-Car naturalistic driving study phase II - Results of the 100-Car field experiment," 2006.

6. C. Robbins and P. Chapman, "How does drivers' visual search change as a function of experience? A systematic review and meta-analysis," Accid. Anal. Prev., vol. 132, p. 105266, Nov. 2019, doi: 10.1016/j.aap.2019.105266.

7. K. Zeeb, A. Buchner, and M. Schrauf, "Is take-over time all that matters? the impact of visual-cognitive load on driver take-over quality after conditionally automated driving," Accid. Anal. Prev., vol. 92, pp. 230-239, Jul. 2016, doi: 10.1016/j.aap.2016.04.002.

8. A. Lotz, N. Russwinkel, and E. Wohlfarth, "Response times and gaze behavior of truck drivers in time critical conditional automated driving take-overs," Transp. Res. Part F Traffic Psychol. Behav., vol. 64, pp. 532-551, Jul. 2019, doi: 10.1016/j.trf.2019.06.008.

9. D. Miller et al., "Distraction Becomes Engagement in Automated Driving," Proc. Hum. Factors Ergon. Soc. Annu. Meet., vol. 59, no. 1, pp. 1676-1680, Sep. 2015, doi: 10.1177/1541931215591362.

10. F. Steinberger, R. Schroeter, and C. N. Watling, "From road distraction to safe driving: Evaluating the effects of boredom and gamification on driving behaviour, physiological arousal, and subjective experience," Comput. Human Behav., vol. 75, pp. 714726, Oct. 2017, doi: 10.1016/j.chb.2017.06.019.

11. T. Vogelpohl, M. Kühn, T. Hummel, and M. Vollrath, "Asleep at the automated wheel-Sleepiness and fatigue during highly automated driving," Accid. Anal. Prev., vol. 126, pp. 70-84, May 2019, doi: 10.1016/j.aap.2018.03.013. 
12. R. Happee, C. Gold, J. Radlmayr, S. Hergeth, and K. Bengler, “Take-over performance in evasive manoeuvres," Accid. Anal. Prev., vol. 106, pp. 211-222, Sep. 2017, doi: 10.1016/j.aap.2017.04.017.

13. [13] M. Körber, A. Cingel, M. Zimmermann, and K. Bengler, "Vigilance Decrement and Passive Fatigue Caused by Monotony in Automated Driving," Procedia Manuf., vol. 3, pp. 2403-2409, Jan. 2015, doi: 10.1016/j.promfg.2015.07.499.

14. T. Arakawa, R. Hibi, and T. aki Fujishiro, "Psychophysical assessment of a driver's mental state in autonomous vehicles," Transp. Res. Part A Policy Pract., vol. 124, pp. 587-610, Jun. 2019, doi: 10.1016/j.tra.2018.05.003.

15. E. A. Morris and E. Guerra, "Are we there yet? Trip duration and mood during travel," Transp. Res. Part F Traffic Psychol. Behav., vol. 33, pp. 38-47, Jul. 2015, doi: 10.1016/j.trf.2015.06.003.

16. A. Malokin, G. Circella, and P. L. Mokhtarian, "How do activities conducted while commuting influence mode choice? Using revealed preference models to inform public transportation advantage and autonomous vehicle scenarios," Transp. Res. Part A Policy Pract., vol. 124, pp. 82-114, Jun. 2019, doi: 10.1016/j.tra.2018.12.015.

17. M. Lakier, L. E. Nacke, T. Igarashi, and D. Vogel, “Cross-car, multiplayer games for semi-autonomous driving," in CHI PLAY 2019 - Proceedings of the Annual Symposium on Computer-Human Interaction in Play, 2019, pp. 467-480, doi: $10.1145 / 3311350.3347166$.

18. N. Broy et al., "A cooperative in-car game for heterogeneous players," in Proceedings of the 3rd International Conference on Automotive User Interfaces and Interactive Vehicular Applications, AutomotiveUI 2011, 2011, pp. 167-174, doi: 10.1145/2381416.2381443.

19. P. E. Paredes et al., "Driving with the Fishes," Proc. ACM Interactive, Mobile, Wearable Ubiquitous Technol., vol. 2, no. 4, pp. 1-21, Dec. 2018, doi: 10.1145/3287062.

20. P. Hock, S. Benedikter, J. Gugenheimer, and E. Rukzio, “CarVR: Enabling in-car virtual reality entertainment," in Conference on Human Factors in Computing Systems - Proceedings, 2017, vol. 2017-May, pp. 4034-4044, doi: 10.1145/3025453.3025665.

21. J. Iskander et al., "From car sickness to autonomous car sickness: A review," Transp. Res. Part F Traffic Psychol. Behav., vol. 62, pp. 716-726, Apr. 2019, doi: 10.1016/j.trf.2019.02.020.

22. [22] M. McGill, J. Williamson, A. Ng, F. Pollick, and S. Brewster, "Challenges in passenger use of mixed reality headsets in cars and other transportation," Virtual Real., vol. 24, no. 4, pp. 583-603, Dec. 2020, doi: 10.1007/s10055-019-00420-x.

23. Audi, "holoride: Virtual Reality meets the real world I audi.com," 2019. [Online]. Available: https://www.audi.com/en/experience-audi/mobility-and-trends/digitalization/holoride-virtual-reality-meets-the-real-world.html. [Accessed: 17-Dec-2020].

24. E. Genty, “Renault SYMBIOZ Demo car: technologies for traveling well-being - Groupe Renault," 2017. [Online]. Available: https://group.renault.com/en/news-on-air/news/renault-symbioz-demo-car-technologies-for-traveling-well-being/. [Accessed: 18-Dec-2020].

25. L. Brunnberg, O. Juhlin, and A. Gustafsson, "Games for passengers - Accounting for motion in location-based applications," in FDG 2009 - 4th International Conference on the Foundations of Digital Games, Proceedings, 2009, pp. 26-33, doi: $10.1145 / 1536513.1536528$.

26. K. Matsumura and D. S. Kirk, “On Active Passengering: Supporting In-Car Experiences,” Proc. ACM Interactive, Mobile, Wearable Ubiquitous Technol., vol. 1, no. 4, pp. 1-23, Jan. 2018, doi: 10.1145/3161176.

27. O. Zuckerman, G. Hoffman, and A. Gal-Oz, “In-car game design for children: Promoting interactions inside and outside the car," Int. J. Child-Computer Interact., vol. 2, no. 4, pp. 109-119, Dec. 2014, doi: 10.1016/j.ijcci.2015.03.001.

28. N. Statt, "Tesla Arcade hands-on: using a Model 3 steering wheel as a game controller - The Verge," 2019. [Online]. Available: https://www.theverge.com/2019/6/18/18684297/tesla-arcade-model-3-hands-on-beach-buggy-racing-2-demo-electric-cars. [Accessed: 17-Dec-2020].

29. S. Schrader, “The New Mercedes-Benz CLA Lets You Play Mario Kart on Its Infotainment Screen," 2019. [Online]. Available: https://www.thedrive.com/news/26761/the-new-mercedes-benz-cla-lets-you-play-mario-kart-on-its-infotainment-screen. [Accessed: 17-Dec-2020].

30. Paul Tassi, “Nintendo Forms Unusual Partnership With Mercedes for 'Mario Kart 8' DLC," 2014. [Online]. Available: https://www.forbes.com/sites/insertcoin/2014/05/29/nintendo-forms-unusual-partnership-with-mercedes-for-mario-kart-8dlc/?sh=4836785b1a67. [Accessed: 17-Dec-2020].

31. CNN, “Amazon's Zoox reveals its robotaxi - CNN," CNN News, 2020. [Online]. Available: https://edition.cnn.com/2020/12/14/cars/zoox-amazon-robotaxi/index.html. [Accessed: 17-Dec-2020].

32. GM, "Chevrolet EN-V 2.0 Vehicle Sharing Pilot Program Concludes," GM Corporate Newsroom, 2017. [Online]. Available: https://media.gm.com/media/cn/en/gm/news.detail.html/content/Pages/news/cn/en/2017/May/0505_Chevrolet-EN-V.html. [Accessed: 17-Dec-2020].

33. M. McGill, J. Williamson, A. Ng, F. Pollick, and S. Brewster, "Challenges in passenger use of mixed reality headsets in cars and other transportation," Virtual Real., no. Prince 2014, 2019, doi: 10.1007/s10055-019-00420-x.

34. W. Vlakveld, N. van Nes, J. de Bruin, L. Vissers, and M. van der Kroft, "Situation awareness increases when drivers have more time to take over the wheel in a Level 3 automated car: A simulator study," Transp. Res. Part F Traffic Psychol. Behav., vol. 58, pp. 917-929, Oct. 2018, doi: 10.1016/j.trf.2018.07.025.

35. P. Lindemann, T. Y. Lee, and G. Rigoll, "Supporting Driver Situation Awareness for Autonomous Urban Driving with an Augmented-Reality Windshield Display," in Adjunct Proceedings - 2018 IEEE International Symposium on Mixed and Augmented Reality, ISMAR-Adjunct 2018, 2018, pp. 358-363, doi: 10.1109/ISMAR-Adjunct.2018.00104.

36. R. Lin, L. Ma, and W. Zhang, "An interview study exploring Tesla drivers' behavioural adaptation," Appl. Ergon., vol. 72, pp. 37-47, Oct. 2018, doi: 10.1016/j.apergo.2018.04.006. 
37. BBCNews, “Tesla in fatal California crash was on Autopilot - BBC News," BBC News, 2018. [Online]. Available: https://www.bbc.com/news/world-us-canada-43604440. [Accessed: 17-Dec-2020].

38. A. Lubben, "Self-driving Uber killed a pedestrian as human safety driver watched," 2018. [Online]. Available: https://www.vice.com/en/article/kzxq3y/self-driving-uber-killed-a-pedestrian-as-human-safety-driver-watched. [Accessed: 17Dec-2020].

39. S. A. Cohen and D. Hopkins, "Autonomous vehicles and the future of urban tourism," Ann. Tour. Res., vol. 74, pp. 33-42, Jan. 2019, doi: 10.1016/j.annals.2018.10.009.

40. E. R. Straub and K. E. Schaefer, "It takes two to Tango: Automated vehicles and human beings do the dance of driving - Four social considerations for policy," Transp. Res. Part A Policy Pract., vol. 122, pp. 173-183, Apr. 2019, doi: 10.1016/j.tra.2018.03.005.

41. W. Chen, X. Zhuang, Z. Cui, and G. Ma, "Drivers' recognition of pedestrian road-crossing intentions: Performance and process," Transp. Res. Part F Traffic Psychol. Behav., vol. 64, pp. 552-564, Jul. 2019, doi: 10.1016/j.trf.2019.07.004.

42. C. Strauch et al., "Real autonomous driving from a passenger's perspective: Two experimental investigations using gaze behaviour and trust ratings in field and simulator," Transp. Res. Part F Traffic Psychol. Behav., vol. 66, pp. 15-28, Oct. 2019, doi: 10.1016/j.trf.2019.08.013.

43. T. Louw and N. Merat, "Are you in the loop? Using gaze dispersion to understand driver visual attention during vehicle automation," Transp. Res. Part C Emerg. Technol., vol. 76, pp. 35-50, Mar. 2017, doi: 10.1016/j.trc.2017.01.001.

44. A. Feldhütter, C. Gold, S. Schneider, and K. Bengler, "How the Duration of Automated Driving Influences Take-Over Performance and Gaze Behavior," in Advances in Ergonomic Design of Systems, Products and Processes, Springer Berlin Heidelberg, 2017, pp. 309-318.

45. E. Peillard, T. Thebaud, J. M. Norrnand, F. Argelaguet, G. Moreau, and A. Lecuyer, "Virtual objects look farther on the sides: The anisotropy of distance perception in virtual reality," in 26th IEEE Conference on Virtual Reality and 3D User Interfaces, VR 2019 - Proceedings, 2019, pp. 227-236, doi: 10.1109/VR.2019.8797826. 